\title{
Demystifying the Retrievability of Cement-retained Implant-supported Restorations
}

\author{
${ }^{1}$ Manesh Lahori, ${ }^{2}$ Lanka Mahesh, ${ }^{3}$ Rahul Nagrath, ${ }^{4}$ Prerna Kaushik
}

\begin{abstract}
The field of implant prostheses has come of age in the postBranemark era. Various research studies are being carried out world over, in order to formulate superior materials, achieve better esthetic outcomes, quicker functional results, minimal treatment complications and maximal comfort to the patient in concern.

Prosthetic reconstruction involving implants may involve screw-retained or cement-retained crowns depending upon the clinician's preference and the clinical situation.

Potential unpredictable biological or mechanical complications that might occur in an implant supported prostheses make retrievability an important point of consideration and so is the treatment planning.

Thus, this case report finds newer means to predict the screw access hole in cases where cement retained prosthesis is indicated.
\end{abstract}

Keywords: Retrievability, Cement retained prosthesis, Screw retained prosthesis, Screw-access hole, Guide holes, Precision implant locating device (PILD).

How to cite this article: Lahori M, Mahesh L, Nagrath $R$, Kaushik P. Demystifying the Retrievability of Cement-retained Implant-supported Restorations. Int J Oral Implantol Clin Res 2014;5(1):29-33.

Source of support: Nil

Conflict of interest: None

\section{INTRODUCTION}

The field of implant prostheses has come of age in the postBranemark era. Various research studies are being carried out world over, in order to formulate superior materials, achieve better esthetic outcomes, quicker functional results, minimal treatment complications and maximal comfort to the patient in concern.

Prosthetic reconstruction involving implants may involve screw-retained or cement-retained crowns depending upon the clinician's preference and the clinical situation.

Potential unpredictable biological or mechanical complications that might occur in an implant supported prostheses

\footnotetext{
${ }^{1}$ Professor, ${ }^{2}$ Private Practitioner, ${ }^{3,4}$ Postgraduate Student

1,3,4 Department of Prosthodontics, Kanti Devi Dental College Mathura, Uttar Pradesh, India

${ }^{2}$ New Delhi, India

Corresponding Author: Manesh Lahori, Professor, Department of Prosthodontics, B-54 SFS flats, Sheikh Sarai, Phase I, New Delhi-110017, India, e-mail: maneshlahori@gmail.com
}

make retrievability an important point of consideration. Thus, the choice of kind of prostheses too is an important consideration.

\section{CEMENT-RETAINED PROSTHESES}

A cement-retained prosthesis has various advantages over screw-retained prostheses that include, superior esthetics, precise occlusion, passive fit, ability to compensate for some implant angulation issues (malposed implants), reduced cost, reduced chair side time, elimination of unesthetic screw access holes, decreased bacterial leakage, greater resistance to porcelain fractures, familiar restorative cementation protocols. Also, the absence of screw to draw inadequately fitting components together with a clamping force would be likely to eliminate strain that the tightening force of screw would introduce into the restoration-implant assembly.

Unfortunately 'retrievability' is a concern with such prostheses. Removing the cement retained prostheses is highly unpredictable due to lack of standardization of techniques and materials used in implant prosthetics and inability to identify the materials used radiographically.

This leads to difficulty and unpredictability in removal, increased treatment time and added costs for patients.

\section{SCREW-RETAINED PROSTHESES}

Adell and coworkers advocate screw retained prostheses since it offers reversibility and more stability and security at the implant-abutment interface.

\section{NEED FOR RETRIEVABILITY}

- Fracture of abutment screws

- Fractured porcelain

- Abutment-screw tightening

- For hygiene

- For repairs

- Retained cement subgingivally may lead to peri-implantitis

- Unacceptable recession of peri-implant gingiva.

The advantages offered by cement-retained prostheses make it a popular choice over screw-retained prostheses. Thus, various methods have been described in literature to provide retrievability of cemented implant supported restorations. Thereby overcoming the only challenge posed by such treatment option. 


\section{VARIOUS APPROACHES FOR RETRIEVING CEMENT-RETAINED PROSTHESES}

\section{Radiographs and Photographs}

Reviewing a radiograph of implant position and estimating the long axis pattern of screw.

Using photograph to record the position of abutment before cementation.

\section{Use of Provisional Cement}

Cements of different retentive capabilities may be used to function as effectively as screws.

But since, they cement metal (of abutment) to metal (of Porcelain Fused to Metal), they become difficult to remove.

\section{Use of Set Screws}

Use of set screws that allow a retrieval screw to be placed in a position where a displacing force can be applied in the direction of the abutment to break the cement seal and allow removal of restoration.

This has the disadvantage of an extra screw on the occlusal surface.

\section{Placement of Stain on Porcelain}

A well defined small ceramic stain on the occlusal surface of a posterior implant-supported restoration in conjunction with a periapical radiograph for evaluating the implant angulation.

This provides a reliable landmark for locating the screw-access opening of the abutment.

But this may be used only in posterior restorations with stain placed in nonesthetic areas.

\section{Secondary Locking Screw}

Use of a secondary locking screw in the restoration for retention and retrievability.

\section{Cylindrical Guide Holes}

Use of cylindrical guide holes in the lingual aspect of implant-supported restorations.

A removing driving placed in these guide holes, when turned, creates a shear force and thereby raises and unseats the restorations.

\section{Vacuum-formed Clear Guides}

Use of vacuum-formed clear retaining screw location guide made on cast. This assists in locating the implant access chamber and abutment retaining screw to facilitate the removal.

\section{CASE REPORTS}

\section{Use of Digital Photographs (Technique 1)}

\section{Procedure}

1. The custom implant abutment was placed over the implant. Then, a periodontal probe was placed at the incisal or occlusal level of the abutment, and facial and incisal view photographs were made (Fig. 1).

2. Periodontal probe was placed vertically at the middle of the space, the tip facing the opposing dentition at the level of the incisal or the occlusal surfaces of the adjacent teeth (Fig. 2).

3. Implant restoration was placed over the abutment and position the periodontal probe horizontally, exactly done in step 1 (Fig. 3).

4. The photographs were printed and kept in the patient's chart.

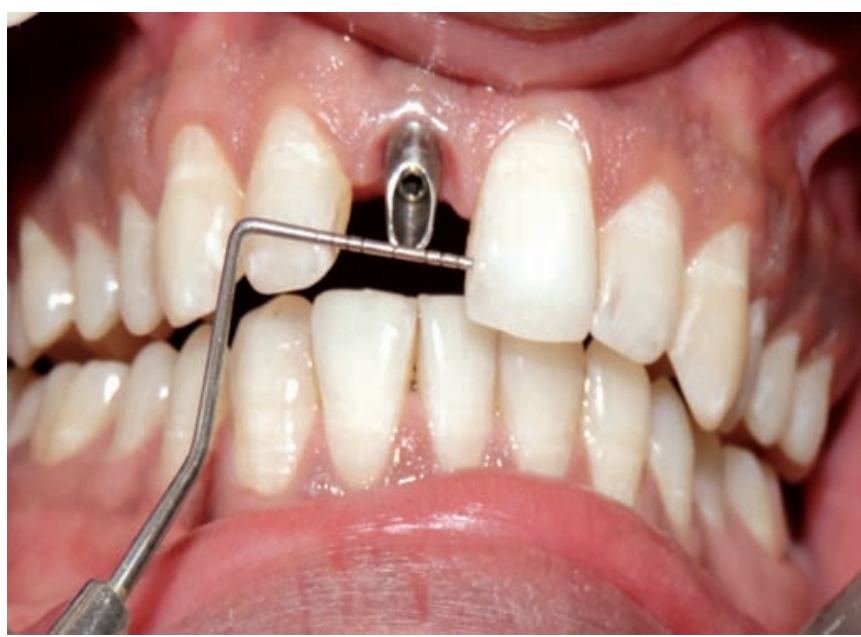

Fig. 1: Facial view of implant custom abutment and horizontally placed periodontal probe at location of incisal top portion of access hole, provides X-axis coordinate of abutment screwaccess opening

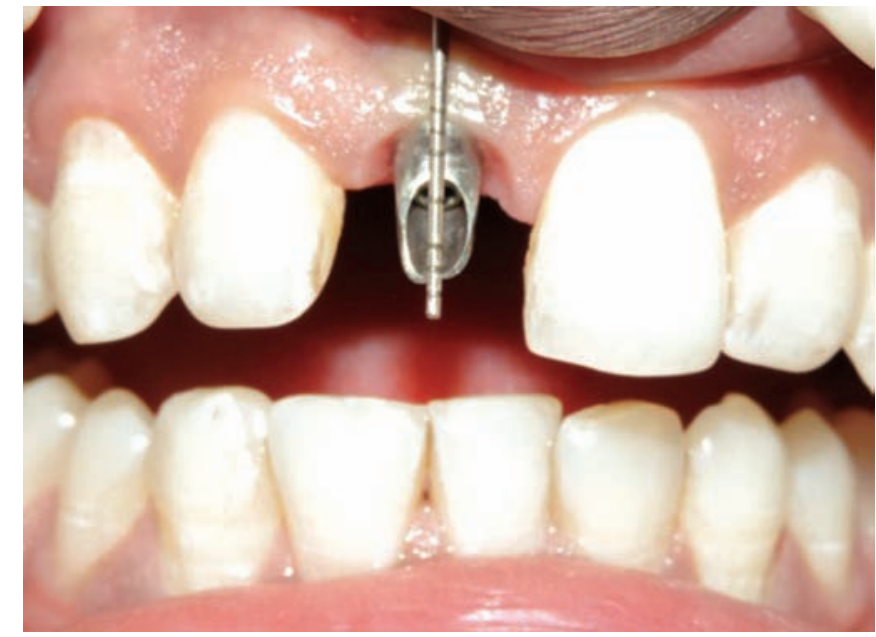

Fig. 2: Implant custom abutment and vertically placed periodontal probe at middle of space between adjacent teeth, provides $\mathrm{Y}$-axis coordinate of abutment screw-access 

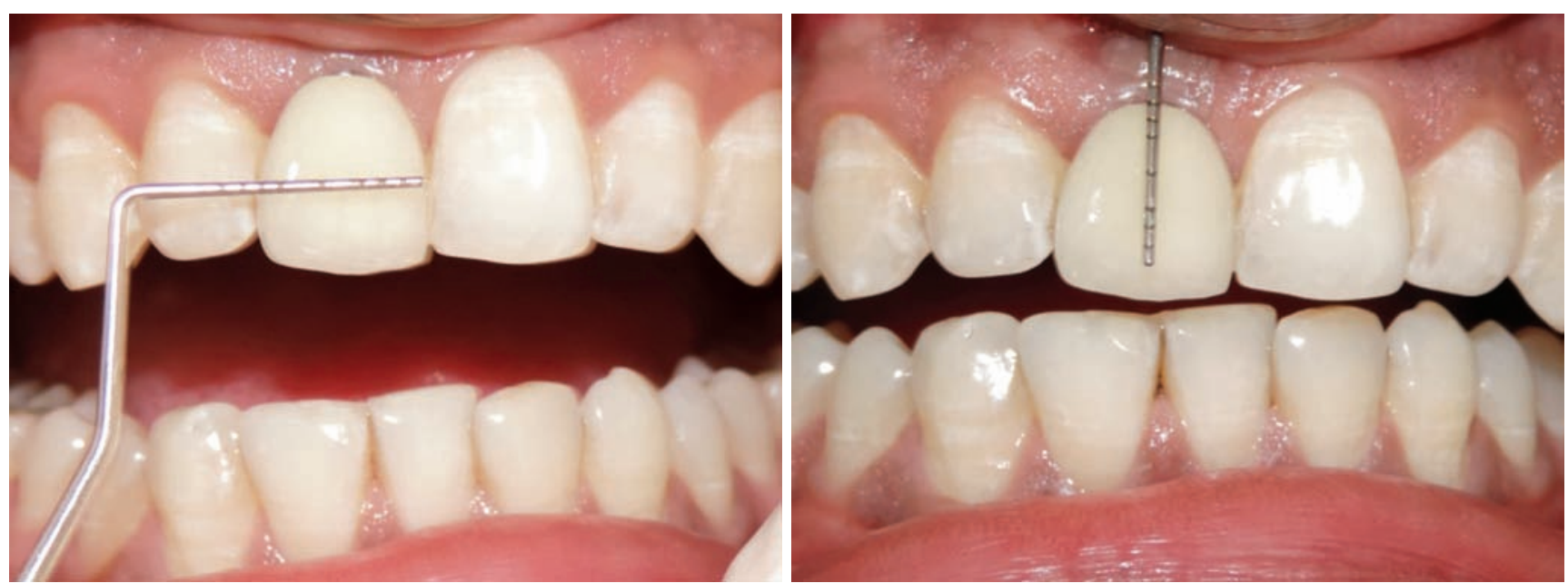

Fig. 3: Cement-retained implant restoration before cementation with probe placed horizontally at location of screw-access opening

5. At a later date, in the event of screw loosening or porcelain fracture, use the photographs to locate the screwaccess hole and drill through the crown in the appropriate position and angulation to access the screw head. The crown may be removed and treated accordingly.

\section{Use of Precision Implant Location Device (Technique 2)}

1. A $25 \times 10 \times 2.7 \mathrm{~mm}$ flat plate was fabricated with custom tray material and light cured for 2 minutes.

2. A hole was prepared that was approximately $2.1 \mathrm{~mm}$ in diameter with a $\# 7$ round carbide bur perpendicular to the center of the plate. Several depressions with a \#2 round carbide bur were made to create retentive elements on the underside of the plate.

3. An appropriate depth probe was inserted into the plate, which acts as a guiding rod, for the given implant system. The plate orifice is designed such that the rod is held perpendicular to the horizontal plane of the plate (Fig. 4).

4. The abutment screw head was located and engaged with the depth probe, then rotate the plate around the guiding rod, which will be used to index the plate so that the rod aligns with the teeth on either side of the

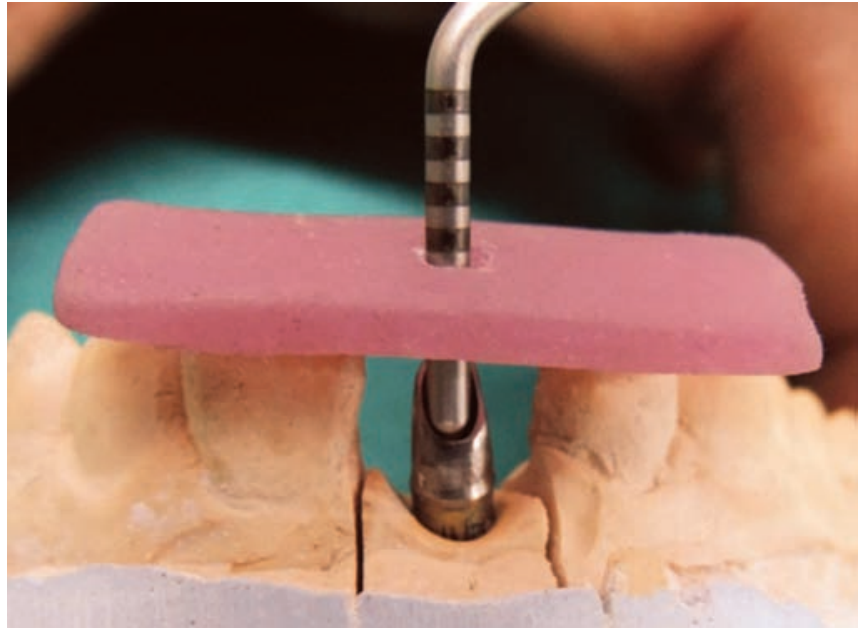

Fig. 4: Use of depth probe to act as a guiding rod

implant restoration. A space of approximately 1 to $2 \mathrm{~mm}$ was maintained between the occlusal surface and the indexing teeth.

5. Impression tray adhesive was applied to the retentive surface of the plate and allowed to dry for 30 seconds.

6. A fast-setting, regular-body occlusal registration material was applied with a fine tip in the space between the inferior side of the plate and the indexing teeth (Fig. 5).
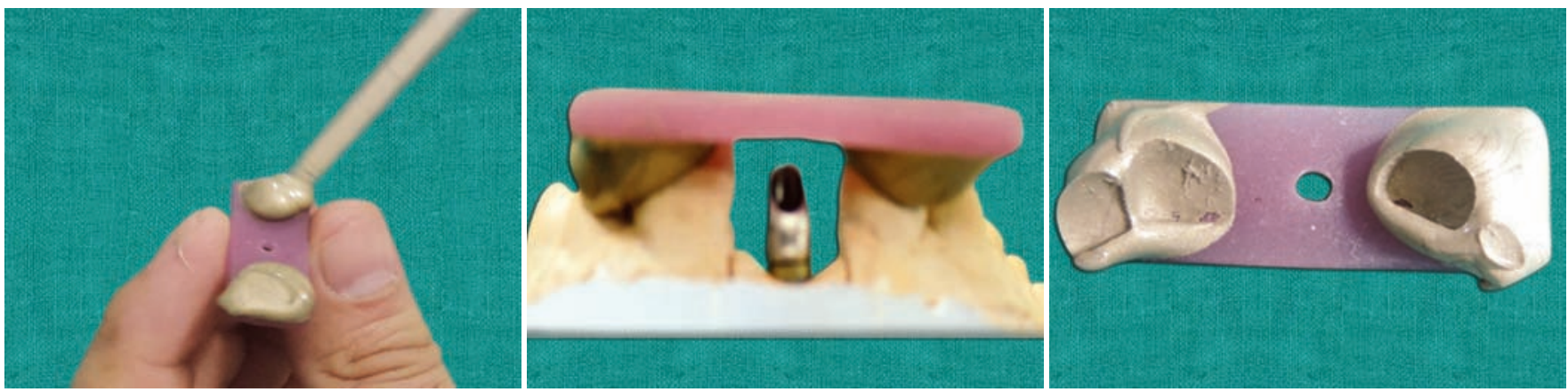

Fig. 5: Fabrication of PILD on cast 


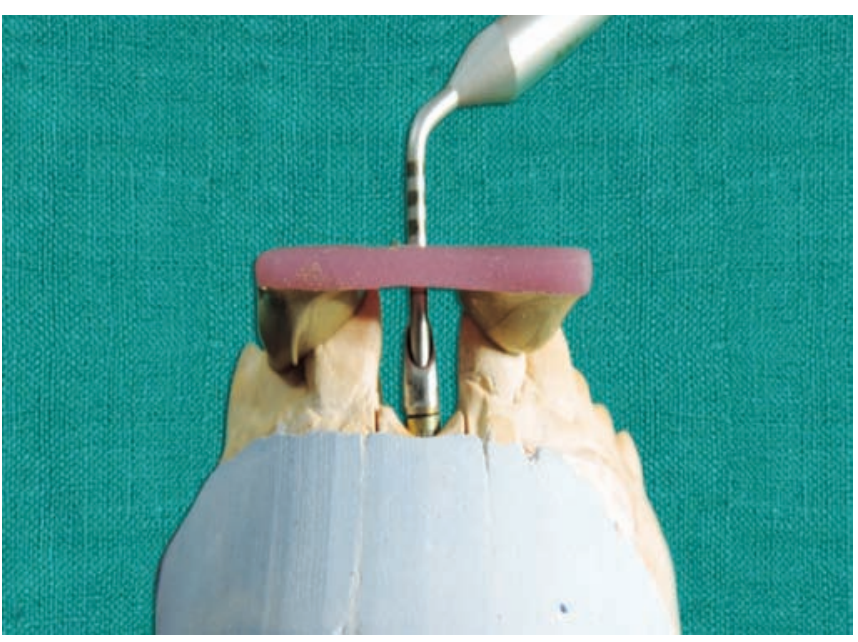

Fig. 6: PILD with depth probe

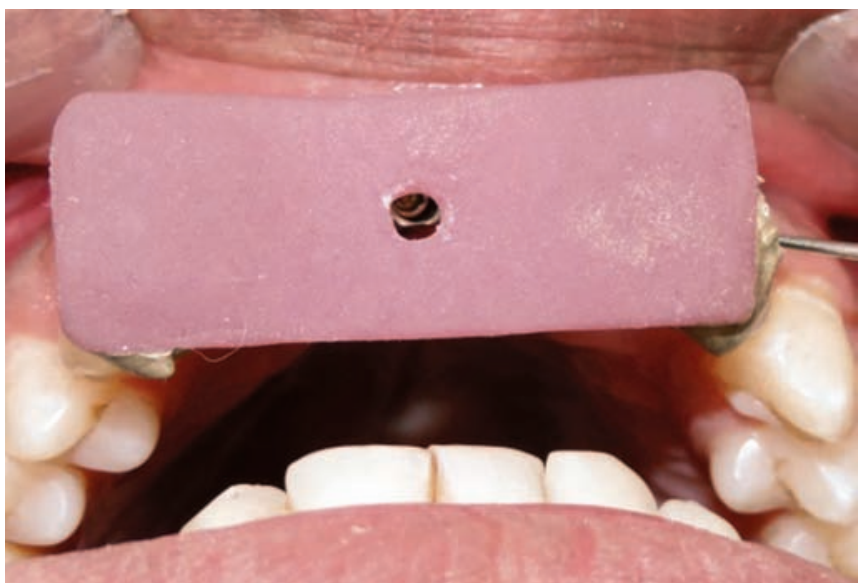

Fig. 8: PILD placed intraorally to check for the position

7. Allow the material to polymerize, remove the PILD from the cast and remove the guiding rod. Ensure that indexing material has not encroached on the implant restoration site. If it has, trim the occlusal registration material, especially at the site that corresponds to the proximal embrasure areas (Fig. 6).

8. Complete the fabrication of the restoration. Place the restoration on the abutment and confirm that the PILD locates easily and is stable. Ensure the guide rod hole of the plate is unobstructed (Figs 7 to 10).

9. Disinfect the PILD and store it with the patient's records.

10. Once the implant crown has been cemented, if the screw needs to be accessed, place the PILD on the adjacent teeth to readily and precisely identify the position of the screw access as well as the axial inclination of the implant.

\section{REFERENCES}

1. Vigolo P, Odont DR, Givani A, Majzoub Z, Cordioli G. Simple device for locating the abutment screw position of a cement-

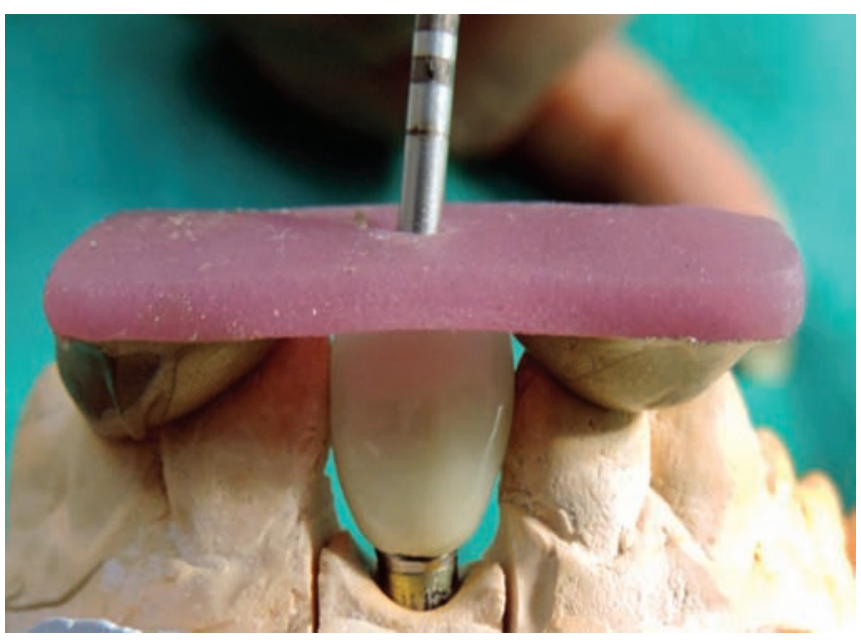

Fig. 7: PILD placed on cast to check for the position with restoration in place

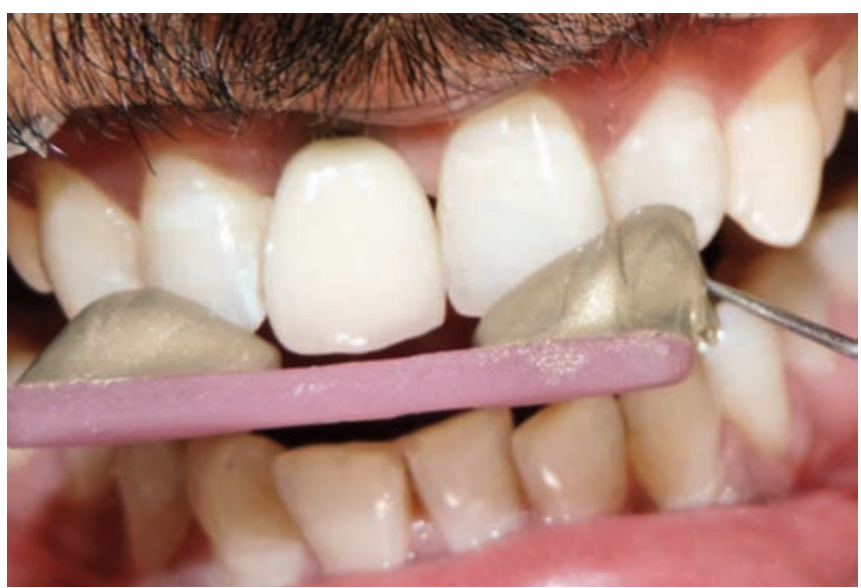

Fig. 9: PILD placed intraorally to check for the position with the restoration in place

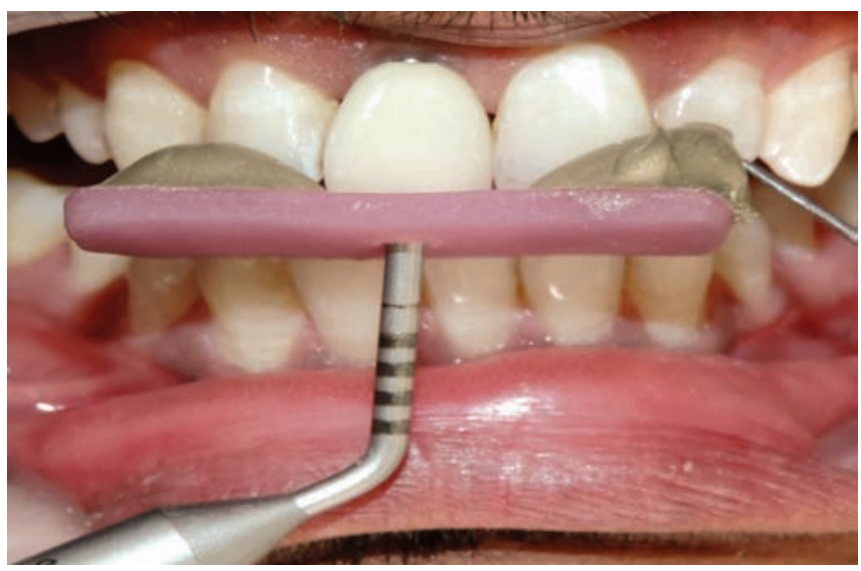

Fig. 10: PILD with the depth probe indicating the precise location of the abutment screw

retained implant restoration. J Prosthet Dent 2013;109: 272-274.

2. Wadhwani C, Chung KH. Cemented versus screw-retained implant-supported single tooth crowns: A 4-year prospective clinical study. Int J Oral Maxillofac Implants 2004;19:2-5.

3. Schoenbaum TR, Chang YY, Klokkevold PR. Screw-access marking: a technique to simplify retrieval of cement-retained implant prostheses (COMPENDIUM March 2013). 
4. Schweitzer DM. A technique for retrievl of cement-retained, implant-supported prosthesis. J Prosthet Dent 2011;106: 131-135.

5. Campbell WF, Herman MW. Choosing between screw retained and cement retained implantcrowns. Inclusive. Spring 2011; 2(2):20-26.

6. Daher T, Morgano SM. The use of digital photographs to locate implant abutment screws for implant supported cement-retained restorations. J Prosthet Dent 2008;100:238-239.

7. Doerr J, Tucson Ariz. Simplified technique for retrieving cemented implant restorations. J Prosthet Dent 2002;88:352-353.

8. Hebel KS, Gajjar RC. Cement-retained versus screw-retained implant restorations: achieving optimum occlusion and esthetics in implant dentistry. J Prosthet Dent 1997;77:28-35.

9. Taylor TD, Agar JR. Twenty years of progress in implant prosthodontics. J Prosthet Dent 2002;88:89-95.

10. Michalakis KX, Hirayama H, Garefis PD. Cement-retained versus screw-retained implant restorations: a critical review. Int J Oral Maxillofac Implants 2003;18:719-728.

11. Chaar MS, Att W, Strub JR. Prosthetic outcome of cementretained implant-supported fixed dental restorations: a systematic review. J Oral Rehabil 2011;38:697-711.
12. Patil PG. A technique for repairing a loosening abutment screw for a cement-retained implant prosthesis. J Prosthodont 2011; 20:652-655.

13. Daher T, Morgano SM. The use of digital photographs to locate implant abutment screws for implant-supported cement retained restorations. J Prosthet Dent 2008;100:238-239.

14. Chee W, Felton DA, Johnson PF, Sullivan DY. Cemented versus screw retained implant prostheses: which is better? Int J Oral Maxillofac Implants 1999;14:137-141.

15. Chee WW, Torbati A, Albouy JP. Retrievable cemented implant restorations. J Prosthodont 1998;7:120-125.

16. Schwedhelm ER, Raigrodski AJ. A technique for locating implant abutment screws of posterior cement-retained metalceramic restorations with ceramic occlusal surfaces. J Prosthet Dent 2006;95:165-167.

17. Clausen GF. The lingual locking screw for implant-retained restorations - aesthetics and retrievability. Aust Prosthodont J 1995;9:17-20.

18. Okamoto M, Minagi S. Technique for removing a cemented superstructure from an implant abutment. J Prosthet Dent 2002;87:241-242.

19. Doerr J. Simplified technique for retrieving cemented implant restorations. J Prosthet Dent 2002;88:352-353. 